\title{
Contrôle et différenciation du lait soumis au traitement U.H.T.
}

\author{
par \\ Bernardo MAIDA et Laura FERRETTI (*)
}

Parmi les éléments capables de définir les caractéristiques du lait mis en commerce (matière grasse, substances sèches, activités enzymatiques, etc.), l'évaluation des protéines (notamment des protéines du sérum) n'a pas encore trouvé une application systématique. Néanmoins, l'emploi de ce paramètre serait souhaitable pour plusieurs raisons. D'abord, il faut considérer que les protéines du lait représentent une source énergétique intégrant celle constituée par la graisse et le lactose ; elles jouent un rôle particulier et unique dans le métabolisme ; c'est en fait de la quantité et de la structure des protéines que dépend la valeur nutritive d'un aliment. Ensuite, une définition de la qualité du lait se basant aussi sur l'évaluation des protéines s'enrichit ultérieurement lorsque les laits analysés sont soumis à des traitements thermiques particulièrement sévères. Dans ce cas, l'évaluation des protéines du sérum constitue un moyen de contrôle de la rationalité du traitement thermique. Il est bien connu que les différentes combinaisons temps-température ou les structures variables des appareils utilisés peuvent donner lieu à différents degrés de dénaturation qui sont décelables dans le lait surtout à travers l'analyse des protéines du sérum.

On peut supposer, donc, que cette analyse serait susceptible, surtout si elle est considérée parmi les paramètres officiels, de limiter l'utilisation de méthodes de traitement peu appropriées et qu'elle entraverait la tendance des techniciens, excessivement soucieux d'obtenir une conservation efficace du lait, à adopter des traitements thermiques plus sévères que ceux qui suffiraient à garantir la stérilisation.

$\mathrm{Au}$ cours de précédentes recherches effectuées par nous [1-2] et par d'autres chercheurs, largement exposées par Burton [3], on avait étudié le comportement des protéines du sérum de lait dans les traitements U.H.T. Dans le présent travail nous illustrerons les

(*) Centrale du Lait de Rome (Directeur : Dr Fausto Bonetti). 
résultats des élaborations faites en vue d'obtenir quelques index synthétiques des effets dénaturants causés par les appareils du type U.H.T.

\section{MATERIEL ET METHODES}

Le lait a été acheté dans les magasins chargés de la vente de ce produit. Il était contenu dans des récipients en carton et il a été analysé pas plus tard que $30 \mathrm{j}$ après son traitement : les échantillons n'ont jamais montré de gélification ou de contamination microbienne.

Les échantillons soumis au traitement thermique indirect (série I) provenaient d'appareils à plaques (système Sordi et Ahlborn, 23 et 1 échantillons respectivement) et d'appareils tubulaires (système Stork et Cherry-Burrel, 6 et 1 échantillons respectivement). Dans le cas du traitement direct (série D) les échantillons provenaient d'appareils Alpura et Alfa-Laval, 4 et 12 échantillons respectivement. L'ensemble de ces échantillons représente la plupart des appareils U.H.T. existant en Italie.

Les contrôles bactériologiques et biochmiques ont été effectués au moyen des procédés illustrés dans une note précédente [2]. Nous ajoutons seulement qu'afin de permettre des élaborations ultérieures, les résultats de l'épreuve de turbidité, d'après Aschaffenburg, ont été exposés dans des termes semi-quantitatifs suivant un classement qui, à partir de 1 (absence de turbidité) arrive jusqu'à 5 (turbidité maximum).

Les valeurs des fractions électrophorétiques, obtenues après acidification du sérum à $\mathrm{pH}$ 4.6 - migration sur acétate de cellulose et lecture au Cromoscan — ont été élaborées de façon à pouvoir calculer les rapports suivants :

$$
\begin{aligned}
& \mathrm{R}_{1}=\frac{\beta \text {-lactoglobuline }}{\alpha \text {-lactalbumine }} \\
& \mathrm{R}_{2}=\frac{\beta \text {-lactoglobuline }+ \text { éventuelles fractions plus rapides }}{\alpha \text {-lactalbumine }} \\
& \mathrm{R}_{3}=\frac{\beta \text {-lactoglobuline }+ \text { éventuelles fractions plus rapides }}{\alpha \text {-lactalbumine }+ \text { fractions lentes }}
\end{aligned}
$$

Les fractions plus rapides de $\beta$-lactoglobuline sont celles du type pré-bêta déjà décrites en électrophorèse sur papier de sérum de lait U.H.T. pressuré [4]. Par la technique de l'électrophorèse sur acétate, employée dans ces recherches, on peut obtenir des fractions pareilles même à partir de sérums acides $(\mathrm{pH} 4.6)$ surtout s'ils proviennent de laits traités avec des systèmes à injection de vapeur. Les fractions qu'on définit lentes sont celles qui occupent la même place que les immuno-globulines. 


\section{RESULTATS}

Afin de simplifier l'analyse des données, laissons de côté celles relatives à chaque échantillon et considérons les valeurs moyennes de chaque série.

Le tableau 1 montre que les valeurs de turbidité et des protéines du sérum sont plus élevées après injection de vapeur (4.63 et - respectivement $-\mathrm{g} 0.36$ p. 100 ) qu'après le traitement indirect (2.74 et - respectivement - g 0.21 p. 100).

Les fractions principales des protéines du sérum présentent un comportement qui est également caractéristique : la $\beta$-lactoglobuline est plus élevée que la $\alpha$-lactalbumine après injection de vapeur (46 p. 100 et - respectivement -33.9 p. 100); inversion des valeurs susdites après traitement indirect $(30.5$ p. 100 - respectivement 46.9 p. 100 ).

Particulièrement intéressant est le comportement des indices $R_{1}, R_{2}$ et $R_{3}$. Ceux-ci, en effet, s'élèvent au-dessus de l'unité dans les laits qui ont subi l'injection de vapeur (respectivement 1.36, 1.62, 1.18 ), tandis qu'ils montrent des valeurs clairement inférieures après le traitement indirect (respectivement $0.66,0.76,0.55$ ) donnant ainsi une mesure quantitative de l'inversion qui s'était produite dans les deux fractions principales.

Les fractions lentes qui occupent la même place que les globulines immunes sont souvent plus élevées dans le lait soumis au traitement indirect et la moyenne de leur valeur en subit les effets. Les fractions rapides en position pré-bêta sont très inconstantes, surtout après réchauffage indirect. Les variations de toutes ces fractions, qui peuvent être en quelque manière liées au traitement subi par le lait, ne présentent pas un comportement systématique et pourtant, elles ne peuvent pas être utilisées pour la mesure des effets du traitement lui-même. Le coefficient de variabilité particulièrement élevé est représentatif de ce comportement (tab. 1).

Dans le choix d'une méthode capable de représenter le plus fidèlement possible les variations des protéines du sérum causées par les traitements U.H.T., se sont révélés de grande utilité non seulement le critère de la variabilité exprimée en pourcentage, mais une comparaison entre les intervalles dans lesquels la moyenne se place avec une probabilité de 99,7 p. 100. Dans le tableau 2 nous avons rapporté les limites maximales et minimales de cet intervalle. Nous avons calculé, en outrê, l'écart existant entre ces intervalles sur la base duquel on a pu observer que les valeurs de quelques épreuves obtenues à partir du lait U.H.T. direct peuvent se superposer aux valeurs de la série U.H.T. indirect (protéines du sérum et fractions rapides) ou être assez proches l'un de l'autre (fractions électrophorétiques restantes et degré de turbidité dans l'épreuve d'Aschaffenburg). Par contre, les valeurs plus différenciées sont celles des rapports $R_{1}, R_{2}$ et en particulier $R_{3}$. 


\section{TABLEAU 1}

Caractéristiques des échantillons examinés

\begin{tabular}{|c|c|c|c|c|}
\hline \multirow{2}{*}{ Analyses } & \multicolumn{2}{|c|}{ Moyenne des valeurs } & \multicolumn{2}{|c|}{$\begin{array}{l}\text { Coefficient de } \\
\text { variabilité }\left({ }^{*}\right)\end{array}$} \\
\hline & $\begin{array}{l}\text { U.H.T } \\
\text { direct }\end{array}$ & $\begin{array}{l}\text { U.H.T. } \\
\text { indirect }\end{array}$ & $\begin{array}{l}\text { U.H.T. } \\
\text { direct }\end{array}$ & $\begin{array}{l}\text { U.H.T. } \\
\text { indirect }\end{array}$ \\
\hline 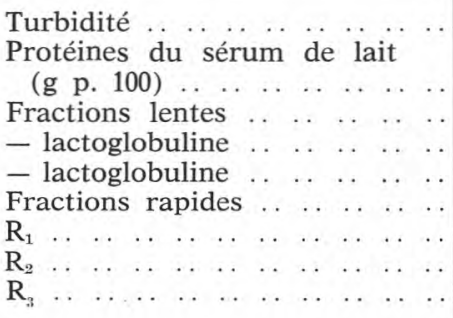 & $\begin{array}{r}4.63 \\
0.30 \\
12.18 \\
33.87 \\
46.00 \\
7.94 \\
1.36 \\
1.62 \\
1.18\end{array}$ & $\begin{array}{r}2.74 \\
\\
0.21 \\
18.30 \\
46.90 \\
30.49 \\
4.48 \\
0.66 \\
0.76 \\
0.55\end{array}$ & $\begin{array}{l}15.5 \\
30.3 \\
34.5 \\
15.1 \\
13.9 \\
78.0 \\
20.0 \\
22.3 \\
18.9\end{array}$ & $\begin{array}{r}43.9 \\
\\
30.9 \\
26.9 \\
15.4 \\
20.2 \\
130.4 \\
30.0 \\
33.4 \\
30.7\end{array}$ \\
\hline
\end{tabular}

(*) C.V. = (déviation standard/moyenne $) \times 100$.

TABLEAU 2

Intervalle des valeurs moyennes $\left(^{*}\right)$

\begin{tabular}{|c|c|c|c|c|c|c|}
\hline \multirow[t]{2}{*}{ Analyses } & \multicolumn{2}{|c|}{$\begin{array}{l}\text { Série } \\
\text { U.H.T. } \\
\text { direct }\end{array}$} & \multicolumn{2}{|c|}{$\begin{array}{c}\text { Série } \\
\text { U.H.T. } \\
\text { indirect }\end{array}$} & \multicolumn{2}{|c|}{$\begin{array}{l}\text { Ecart entre les } \\
\text { intervalles }(* *)\end{array}$} \\
\hline & Max. & Min. & Max. & Min. & Absolu & p. 100 \\
\hline Turbidité & 5.17 & 4.09 & 3.39 & 2.09 & 0.70 & 17 \\
\hline $\begin{array}{l}\text { Protéines du sérum de lait } \\
\quad(\mathrm{g} \text { p. } 100)\end{array}$ & & 0.23 & 0.24 & 0.17 & -0.01 & -4 \\
\hline Fractions lentes $\ldots$ & 13.23 & 11.13 & 20.95 & 15.65 & 2.42 & 15 \\
\hline$\alpha$-lactoglobuline . . . . . . . . & 37.71 & 30.03 & 50.80 & 43.00 & 5.29 & 12 \\
\hline$\beta$-lactoglobuline . . . . . & 50.79 & 41.21 & 33.81 & 27.17 & 7.40 & 18 \\
\hline Fractions rapides .. & 12.58 & 3.30 & 7.63 & 1.33 & -4.33 & -57 \\
\hline $\begin{array}{llllllllllll}\mathbf{R}_{1} & \ldots & \ldots & \ldots & \ldots & \ldots & \ldots & \ldots & \ldots & \ldots & .\end{array}$ & 1.56 & 1.15 & 0.77 & 0.55 & 0.38 & 33 \\
\hline $\begin{array}{llllllllll}\mathbf{R}_{2} & \ldots & \ldots & \ldots & \ldots & \ldots & \ldots & \ldots & \ldots\end{array}$ & 1.90 & 1.35 & 0.90 & 0.62 & 0.45 & 33 \\
\hline $\begin{array}{llllllllll}\mathrm{R}_{3} & \ldots & \ldots & \ldots & \ldots & \ldots & \ldots & \ldots & \ldots & \ldots\end{array}$ & 1.35 & 1.02 & 0.64 & 0.46 & 0.38 & 37 \\
\hline
\end{tabular}

(*) Cet intervalle est égal à $\mathrm{m} \pm 3 \mathrm{~s}_{\mathrm{m}}$ ( $\mathrm{s}_{\mathrm{m}}$ est l'erreur standard de la moyenne).

${ }^{(* *)}$ Ecart entre les intervalles = limite minimale de la série avec valeurs majeures - limite maximale de la série avec valeurs mineures. 


\section{CONCLUSIONS}

Il s'ensuit des élaborations ci-dessus exposées que les rapports $R_{1}, R_{2}$ et $R_{3}$ donnent des résultats qui reflètent les caractéristiques du traitement subi par le lait d'une manière plus fidèle que ne le font les autres paramètres comparés entre eux : ce qui est mis en évidence par l'écart net des moyennes et des limites d'erreur. On peut aboutir à une conclusion similaire en calculant la signification statistique des différences entre les moyennes des séries D et I. En effet, la signification est plus élevée si les différences entre traitements U.H.T. direct et indirect sont révélées au moyen des rapports que nous avons proposés : les valeurs de $t$, qui sont significatives à un niveau supérieur à 1 p. 1000 , ont été $-10.09,-9.55$ et -10.95 respectivement pour $R_{1}, R_{2}$ et $R_{3}$ vis-à-vis de -5.76 et -3.85 respectivement pour le test de turbidité et la tenẹr en protéines du sérum.

L'inversion du rapport $\beta$-lactoglobuline/ $\alpha$-lactalbumine au cours du traitement U.H.T. indirect et la mesure du phénomène par l'un des indices proposés offrent la possibilité de reconnaître le traitement subi par le lait. Mais ce qui importe plus que cette individualisation " a posteriori ", c'est l'indication d'une limite objective audelà de laquelle il n'est pas opportun de pousser la dénaturation des protéines du sérum. Cela peut être atteint par l'emploi approprié d'appareils à injection de vapeur ou de type analogue. En ce qui concerne, au contraire, les traitements indirects, même si l'on n'exclut pas qu'on puisse mettre au point des appareils capables d'aboutir à des résultats meilleurs, il est évident que certains stocks de lait, qui ont subi ce traitement, prennent les caractéristiques du lait autoclavé : à peu près 23 p. 100 de nos échantillons examinés se sont révélés parfaitement limpides après l'épreuve d'Aschaffenburg, tandis que cela ne s'est jamais produit dans les échantillons provenant d'installations à injection de vapeur. Il est évident, par conséquent, que dans le cas du traitement indirect il serait opportun d'obtenir au moins un test de turbidité positif.

Puisque nous nous trouvons en face de types de lait stérile différents, il serait à ce sujet-là opportun de définir avec objectivité cette variété dans le but aussi d'établir une qualification d'ordre commercial qui tienne compte des charges plus élevées nécessaires pour obtenir un aliment de qualité meilleure : coût des appareils et de leur entretien, sélection rigoureuse du lait cru, emploi de personnel qualifié.

Les considérations précédentes nous amènent au classement suivant du lait ainsi dit stérile :

1) lait ultra-pasteurisé, généralement traité dans les installalations D.U.H.T. (direct ultra high temperature). 
Il est caractérisé par le test de turbidité positif et par les rapports électrophorétiques tels qu'ils se révèlent dans le lait cru ou pasteurisé $\left(R_{1}, R_{2}, R_{3} \geqslant 1\right)$;

2) lait surchauffé, généralement traité dans les installations I.U.H.T. (indirect ultra-high temperature). On peut exiger que le test de turbidité résultant soit positif. Les rapports électrophorétiques sont renversés $\left(R_{1}, R_{2}, R_{3}<1\right)$;

3) lait cuit, généralement traité dans les appareils de type autoclave (autoclaved milk) : ce lait présente un test de turbidité négatif et des fractions électrophorétiques qui ne peuvent pas être différenciées du tout ou seulement en partie (lorsque la différenciation est possible $R_{1}, R_{2}, R_{3}<1$ ).

\section{Rés u m é}

La nécessité d'évaluer le taux des protéines du sérum est considérée afin de définir la qualité des laits qui ont subi le traitement thermique U.H.T.

Des élaborations ont été effectuées pour mesurer les effets dénaturants produits par différentes installations du type U.H.T. (traitement thermique direct et indirect).

Certains rapports existant entre les fractions électrophorétiques se placent au-dessus de l'unité dans les laits qui ont subi l'injection de vapeur, tandis qu'ils montrent des valeurs inférieures dans les laits à traitement indirect.

L'écart net entre les moyennes, statistiquement significatif, confirme qu'une différence systématique existe entre les deux types de traitement employés et suggère la possibilité de mesurer l'effet dénaturant à travers l'emploi des rapports proposés.

Par ces fondements nous aboutissons à une classification du lait dit stérile.

\section{S u m m a r y}

The necessity of evaluating the rate of the proteins of the whey is considered to give a definition of the quality of the ultra-high temperature processed milk.

Some data were worked out in order to measure the denaturant effects produced by the equipments like U.H.T. (direct and indirect heat treatment).

Some ratios existing among the electrophoretic fractions exceed the unit in the milk submitted to steam injection, while they store lower values in the indirect processed milk.

The important difference between the average data statistically significative reveals that a systematical variation exists between the two techniques and it suggests, moreover the possibility of measuring the denaturating effect by means of the above mentioned ratios.

On these bases we wont to do one grading of steril milks. 


\section{Bibliographie}

[1] Maida (B.), Ferretti (L.), Stinchi (S.) (1968). - Primi risultati di una indagine sui latti a lunga conservazione : Nota $1 a-\mathrm{Su}$ alcune caratteristiche microbiologiche e biochimiche. Il Latte, 42, 874-875.

[2] Maida (B.), Ferretti (L.) (1968). - Primi risultati di una indagine sui latti a lunga conservazione : Nota $2 a$ - Comportamento eletroforetico delle sieroproteine. Il Latte, 42, 876-878.

[3] Burton (H.) (1969). - Ultra high temperature processed milk. Dairy Science Abstracts, 31, 287-297.

[4] MaIDA (B.) (1967). - Fraction électrophorétique en position pré-bêta dans le sérum de lait chauffé à haute température. Le Lait, 48, 261-266.

Reçu pour publication en septembre 1970. 